\title{
Precise asymptotics - a general approach
}

Allan Gut and Josef Steinebach

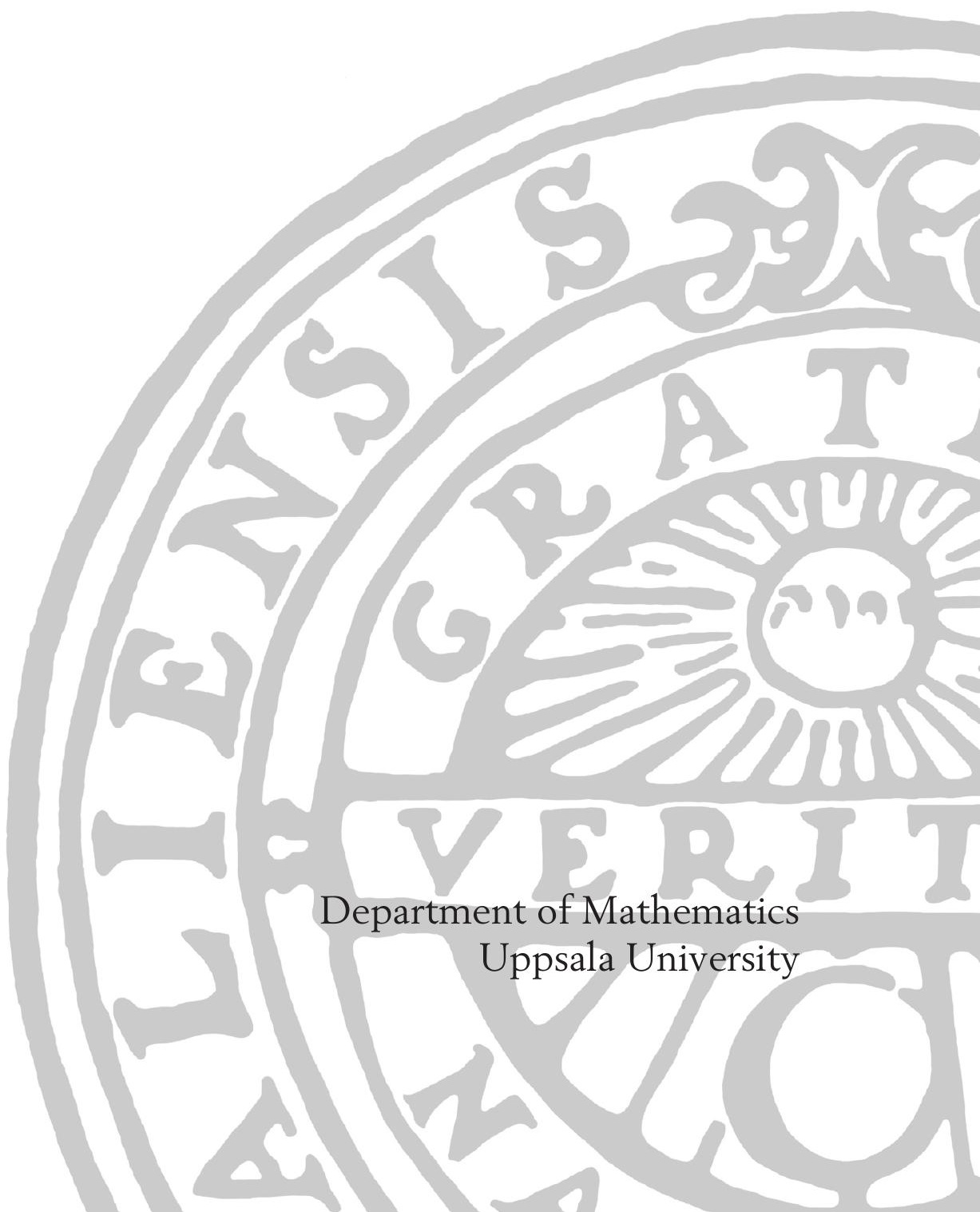





\title{
Precise asymptotics—a general approach
}

\author{
Allan Gut \\ Uppsala University University of Cologne \\ Josef Steinebach
}

\begin{abstract}
The legendary 1947-paper by Hsu and Robbins, in which the authors introduced the concept of "complete convergence", generated a series of papers culminating in the like-wise famous Baum-Katz 1965-theorem, which provided necessary and sufficient conditions for the convergence of the series $\sum_{n=1}^{\infty} n^{r / p-2} P\left(\left|S_{n}\right| \geq \varepsilon n^{1 / p}\right)$ for suitable values of $r$ and $p$, in which $S_{n}$ denotes the $n$-th partial sum of an i.i.d. sequence. Heyde followed up the topic in his 1975paper in that he investigated the rate at which such sums tend to infinity as $\varepsilon \searrow 0$ (for the case $r=2$ and $p=1$ ). The remaining cases have been taken care of later under the heading "precise asymptotics". An abundance of papers have since then appeared with various extensions and modifications of the i.i.d.-setting. The aim of the present paper is to show that the basis for the proof is essentially the same throughout, and to collect a number of examples. We close by mentioning that Klesov, in 1994, initiated work on rates in the sense that he determined the rate, as $\varepsilon \searrow 0$, at which the discrepancy between such sums and their "Baum-Katz limit" converges to a nontrivial quantity for Heyde's theorem. His result has recently been extended to the complete set of $r$ - and $p$-values by the present authors.
\end{abstract}

\section{Introduction}

In their seminal paper [16] Hsu and Robbins introduced the concept of complete convergence and proved that the sequence of arithmetic means of independent, identically distributed (i.i.d.) random variables converges completely to the expected value of the variables, provided their variance is finite. The necessity was proved somewhat later by Erdős [5, 6]. Formally:

Theorem 1.1 Let $X, X_{1}, X_{2}, \ldots$ be i.i.d. random variables, and set $S_{n}=\sum_{k=1}^{n} X_{k}, n \geq 1$. If $E X^{2}<\infty$ and $E X=0$, then

$$
\sum_{n=1}^{\infty} P\left(\left|S_{n}\right|>n \varepsilon\right)<\infty \quad \text { for all } \varepsilon>0
$$

Conversely, if the sum is finite for some $\varepsilon>0$, then $E X=0, E X^{2}<\infty$, and the sum is finite for all $\varepsilon>0$.

Whereas convergence is a qualitative result in the sense that it tells us that convergence holds, a rate result is a quantitative result in the sense that it tells us how fast convergence is obtained, and how large a sample must be for a certain precision.

In addition to being a result on another kind of convergence, Theorem 1.1 can be viewed as a result on the rate of convergence in the law of large numbers. Namely, not only do the terms $P\left(\left|S_{n}\right|>\varepsilon n\right)$ have to tend to 0 , the sum of them has to converge, which is a little more.

This idea can be pursued further. Following is a more general result linking integrability of the summands to the rate of convergence in the law of large numbers. However, the proof is more technical, since dealing with moment inequalities for sums of an arbitrary order is much more messy than adding variances.

The following result (and more) was proved by Baum and Katz [1].

AMS 2000 subject classifications. Primary 60F05, 60F15, 60G50, 60K05; Secondary 62G10, 62 G20.

Keywords and phrases. Law of large numbers, Hsu-Robbins, Baum-Katz, convergence rates.

Abbreviated title. Precise asymptotics.

Date. October 26, 2011 
Theorem 1.2 Let $r>0,0<p<2$ and $r \geq p$. Suppose that $X, X_{1}, X_{2}, \ldots$ are i.i.d. random variables with $E|X|^{r}<\infty$ and, if $r \geq 1, E X=0$, and set $S_{n}=\sum_{k=1}^{n} X_{k}, n \geq 1$. Then

$$
\sum_{n=1}^{\infty} n^{r / p-2} P\left(\left|S_{n}\right| \geq \varepsilon n^{1 / p}\right)<\infty \quad \text { for all } \varepsilon>0 .
$$

Conversely, if the sum is finite for some $\varepsilon>0$, then $E|X|^{r}<\infty$ and, if $r \geq 1, E X=0$. In particular, the conclusion then holds for all $\varepsilon>0$.

REMARK 1.1 For $r=2$ and $p=1$ the result reduces to the theorem of Hsu and Robbins [16] (sufficiency) and Erdős [5, 6] (necessity). For $r=p=1$ we rediscover the famous theorem of Spitzer [29]. For $r>0$ and $p=1$ the result was earlier proved by Katz; see [19].

Another way to view these sums is to note that the sums tend to infinity as $\varepsilon \searrow 0$. It might therefore be of interest to find the rate at which this occurs. This amounts to finding appropriate normalizations in terms of functions of $\varepsilon$ that yield nontrivial limits. Toward this end Heyde [15] proved that

$$
\lim _{\varepsilon \searrow 0} \varepsilon^{2} \sum_{n=1}^{\infty} P\left(\left|S_{n}\right| \geq \varepsilon n\right)=E X^{2}
$$

whenever $E X=0$ and $E X^{2}<\infty$. Remaining values of $r$ and $p$ have later been taken care of in $[2,28,9]$. For ease of reference we state those results here.

Theorem 1.3 Let $r \geq 2$ and $0<p<2$. Suppose that $X, X_{1}, X_{2}, \ldots$ are i.i.d. random variables with $E X=0, E X^{2}=\sigma^{2}>0$ and $E|X|^{r}<\infty$, and set $S_{n}=\sum_{k=1}^{n} X_{k}, n \geq 1$. Then

$$
\lim _{\varepsilon \searrow 0} \varepsilon^{2(r-p) /(2-p)} \sum_{n=1}^{\infty} n^{r / p-2} P\left(\left|S_{n}\right| \geq \varepsilon n^{1 / p}\right)=\frac{p}{r-p} E|Y|^{2(r-p) /(2-p)},
$$

where $Y$ is normal with mean 0 and variance $\sigma^{2}$.

Theorem 1.4 Suppose that $X, X_{1}, X_{2}, \ldots$ are i.i.d. random variables with mean 0 that belong to the normal domain of attraction of a nondegenerate stable law $G$ with characteristic exponent $\alpha \in(1,2]$, and set $S_{n}=\sum_{k=1}^{n} X_{k}, n \geq 1$. Then, for $1 \leq p<r<\alpha$,

$$
\lim _{\varepsilon \searrow 0} \varepsilon^{(\alpha p /(\alpha-p))(r / p-1)} \sum_{n=1}^{\infty} n^{r / p-2} P\left(\left|S_{n}\right| \geq \varepsilon n^{1 / p}\right)=\frac{p}{r-p} E|Z|^{(\alpha p /(\alpha-p))(r / p-1)},
$$

where $Z$ is a random variable with distribution $G$.

Theorem 1.5 Suppose that $X, X_{1}, X_{2}, \ldots$ are i.i.d. random variables with mean 0 that belong to the domain of attraction of a nondegenerate stable law $G$ with characteristic exponent $\alpha \in(1,2]$, and set $S_{n}=\sum_{k=1}^{n} X_{k}, n \geq 1$. Then, for $1 \leq p<\alpha \leq 2$,

$$
\lim _{\varepsilon \searrow 0} \frac{1}{-\log \varepsilon} \sum_{n=1}^{\infty} \frac{1}{n} P\left(\left|S_{n}\right| \geq \varepsilon n^{1 / p}\right)=\frac{\alpha p}{\alpha-p} .
$$

In particular, if $\operatorname{Var} X=\sigma^{2}<\infty$, then the limit exists and equals $2 p /(2-p)$.

In view of the central limit theorem, there cannot be any analog for $p=2$. However, by replacing $n^{1 / p}$ by $\sqrt{n \log n}$ or $\sqrt{n \log \log n}$, analogous results have been given in [10].

In Section 2 we begin by describing in words the general pattern of the proof(s), after which we, in the second subsection, provide the technical details of our general results. Section 3 contains a list of examples that we have encountered in about 75 papers. More precisely, a first list contains variations of the pure i.i.d.-setting, and a second list contains rate results for other quantities than (weighted) sums of (i.i.d.) random variables. In a final section we present an introduction to rate results, where, to the best of our knowledge, only the i.i.d.-case has been considered (so far). 


\section{Some general result on precise asymptotics}

In this section, more precisely, in Subsection 2.2, we provide, as stated in the heading, some general results on precise asymptotics. However, in order to motivate this study we first present, in Subsection 2.1, the general procedure of the proofs, that is, we isolate the skeleton of the general pattern.

\subsection{The general pattern of the proofs}

The procedure of the proofs in the area can be divided into the following two steps, the last of which frequently is divided into two pieces.

\section{Step I}

One proves the desired result for the appropriate limit distribution, typically standard normal or stable. This step amounts to some purely computational work, and is performed once and for all, since if the limit is normal, say, well, then it is normal irrespectively of the assumptions of the actual sequence at hand concerning independence or identical distribution, martingale difference, moving average, etcetera.

\section{Step II}

One proves the result for the actual sequence under consideration, that is, one shows that the discrepancy between the actual sequence and the limit is asymptotically negligible. This step is typically divided into two parts, for which the sums are truncated at some "convenient point $N=N(\varepsilon, M)$ to be decided upon later".

\section{Step IIa}

Since the difference between the original probabilities, say $P\left(\left|S_{n}\right|>\varepsilon n^{1 / p}\right)$, and the limiting probability, e.g. $P\left(|Y| \geq \varepsilon n^{1 / p-1 / 2}\right)$, tends to zero, it follows that a properly weighted average (that is, summing from 1 to $N$ and normalizing) does so too (cf. e.g. [8], Lemma A.6.1.)

\section{Step IIb}

It remains to establish the same for the difference of the tails, that is for the sums from $N$ to infinity. This step frequently uses the fact that the tails themselves are small, so it suffices, by the triangle inequality, to prove that the corresponding sums themselves are small.

In the sequel we stick to Step IIb in one go by assuming that there exists a common bound on the tails of the process under investigation and of the limit random variable (assumption (A.2) below). Then it is enough to show that the (weighted) sum from $N$ to infinity of this bound tends to zero.

This concludes the proof.

Reviewing the procedure we thus find that

1. We need a limit theorem, such as the central limit theorem;

2. We must compute the desired conclusion for the limiting random variable;

3. The properly normalized sum up to some suitable point of truncation of the difference between the original tail probabilities and those for the limiting random variable converges to zero (this is always "immediate");

4. The appropriate quantity for a common bound on the tails of the limiting random variable and the actual process must converge to zero. 
The main point of our paper is to elucidate the fact that it is in reality only the very last step that requires some work, the others can be taken over from earlier papers (once the limit theorem exists).

In the following subsection we provide a general setup for results on precise asymptotics, in such a manner that any new result is proved by verifying that the basic assumptions below are fulfilled. Later, in Section 3 we shall do so for some of the 14 examples mentioned in the first list there.

\subsection{Technical details}

Assume that the sequence $\left\{Z_{n}\right\}_{n=1,2, \ldots}$ of real-valued random variables satisfies the following assumptions:

(A.1) For some $0<\alpha \leq 2,\left|Z_{n}\right| / n^{1 / \alpha} \stackrel{d}{\rightarrow}|Z|$ as $n \rightarrow \infty$, where $|Z|$ has a continuous distribution function or, equivalently, where $\Psi:[0, \infty) \rightarrow[0,1], \Psi(x)=P(|Z| \geq x)$, is continuous.

(A.2) For some $0<r<\alpha$ there is a positive constant $C$ such that, for all $n \geq 1$ and $x>0$,

$$
\max \left\{P(|Z| \geq x), P\left(\left|Z_{n}\right| / n^{1 / \alpha} \geq x\right)\right\} \leq C x^{-r} .
$$

REMARK 2.1 Note that, by Markov's inequality, a sufficient condition for (A.2) to hold is

(A.2') For some $0<r<\alpha$, there is a positive constant $C$ such that, for all $n \geq 1$,

$$
\max \left\{E|Z|^{r}, E\left(\left|Z_{n}\right| / n^{1 / \alpha}\right)^{r}\right\} \leq C .
$$

Theorem 2.1 Under Assumptions (A.1)-(A.2) and $\left.E\right|^{(\alpha p /(\alpha-p))(r / p-1)}<\infty$, for some $0<$ $p<r(<\alpha)$, we have

$$
\lim _{\varepsilon \searrow 0} \varepsilon^{(\alpha p /(\alpha-p))(r / p-1)} \sum_{n=1}^{\infty} n^{r / p-2} P\left(\left|Z_{n}\right| \geq \varepsilon n^{1 / p}\right)=\frac{p}{r-p} E|Z|^{(\alpha p /(\alpha-p))(r / p-1)} .
$$

We note for later use that the exponent of $\varepsilon$ in $(2.1)$ can be rewritten as $\alpha(r-p) /(\alpha-p)$.

REMARK 2.2 The moment assumption of Theorem 2.1 is satisfied if, for some constant $C$,

$$
P(|Z| \geq x) \leq C x^{-\alpha} \quad \text { for all } \quad x>0
$$

e.g., if $Z$ has a stable distribution with index $\alpha$, since in this case, $E|Z|^{\beta}<\infty$ for all $0 \leq \beta<\alpha$, in particular for $\beta=\alpha(r-p) /(\alpha-p)$.

We also mention that, in the sequel, $C$ denotes a positive constant, which may change between appearances.

For the proof of Theorem 2.1 we follow the arguments in Subsection 2.1 (see also the proof of Theorem 1 in Gut and Spătaru [9]). That is, in a first step we show that the asymptotic (2.1) holds if $\left|Z_{n}\right|$ is replaced by $n^{1 / \alpha}|Z|$, and in a second step it is verified that the discrepancy incurred by this replacement is asymptotically negligible.

Proposition 2.1 Under Assumption (A.1) and $E|Z|^{(\alpha p /(\alpha-p))(r / p-1)}<\infty$, for some $0<p<r$ $(<\alpha)$, we have

$$
\lim _{\varepsilon \searrow 0} \varepsilon^{(\alpha p /(\alpha-p))(r / p-1)} \sum_{n=1}^{\infty} n^{r / p-2} P\left(|Z| \geq \varepsilon n^{1 / p-1 / \alpha}\right)=\frac{p}{r-p} E|Z|^{(\alpha p /(\alpha-p))(r / p-1)} .
$$

Proof. We first assume that $r / p \leq 2$, i.e., $p \geq r / 2$. Then,

$$
\int_{1}^{\infty} x^{r / p-2} \Psi\left(\varepsilon x^{1 / p-1 / \alpha}\right) d x \leq \sum_{n=1}^{\infty} n^{r / p-2} \Psi\left(\varepsilon n^{1 / p-1 / \alpha}\right) \leq \int_{0}^{\infty} x^{r / p-2} \Psi\left(\varepsilon x^{1 / p-1 / \alpha}\right) d x
$$


The change of variable $y=\varepsilon x^{1 / p-1 / \alpha},(2.3)$ yields

$$
\begin{aligned}
\frac{\alpha p}{\alpha-p} \int_{\varepsilon}^{\infty} y^{(\alpha p /(\alpha-p))(r / p-1)-1} \Psi(y) d y & \leq \varepsilon^{(\alpha p /(\alpha-p))(r / p-1)} \sum_{n=1}^{\infty} n^{r / p-2} \Psi\left(\varepsilon n^{1 / p-1 / \alpha}\right) \\
& \leq \frac{\alpha p}{\alpha-p} \int_{0}^{\infty} y^{(\alpha p /(\alpha-p))(r / p-1)-1} \Psi(y) d y
\end{aligned}
$$

and since

$$
\frac{\alpha p}{\alpha-p} \int_{0}^{\infty} y^{(\alpha p /(\alpha-p))(r / p-1)-1} \Psi(y) d y=\frac{p}{r-p} E|Z|^{(\alpha p /(\alpha-p))(r / p-1)},
$$

(2.2) is immediate from $(2.4)$.

In case $r / p>2$ we need some slight modifications of the above arguments, which will briefly be indicated for the upper bound only. The proof for the lower bound works in an analogous manner.

Note that the upper bound in (2.3) can now be replaced by

$$
1+\int_{1}^{\infty}(x+1)^{r / p-2} \Psi\left(\varepsilon x^{1 / p-1 / \alpha}\right) d x
$$

after which the same change of variable $y=\varepsilon x^{1 / p-1 / \alpha}$ as above yields the upper estimate

$$
\varepsilon^{(\alpha p /(\alpha-p))(r / p-1)}+\frac{\alpha p}{\alpha-p} \int_{\varepsilon}^{\infty} y^{(\alpha p /(\alpha-p))(r / p-1)-1}\left(1+r_{\varepsilon}(y)\right)^{r / p-2} \Psi(y) d y,
$$

where $r_{\varepsilon}(y)=\left(1+(\varepsilon / y)^{\alpha p /(\alpha-p)}\right)^{r / p-2}$ is bounded on $[\varepsilon, \infty)$ and converges to 1 as $\varepsilon \rightarrow 0$. So, the same argument as above in combination with Lebesgue's dominated convergence theorem yields the desired upper bound here, too.

A similar argument applied to the modified lower bound completes the proof.

Proposition 2.2 Under Assumptions (A.1) and (A.2) we have, for all $0<p<r(<\alpha)$,

$$
\lim _{\varepsilon \searrow 0} \varepsilon^{(\alpha p /(\alpha-p))(r / p-1)} \sum_{n=1}^{\infty} n^{r / p-2}\left|P\left(\left|Z_{n}\right| \geq \varepsilon n^{1 / p}\right)-P\left(|Z| \geq \varepsilon n^{1 / p-1 / \alpha}\right)\right|=0 .
$$

Proof. For $0<\varepsilon \leq 1$ and $M \geq 1$ set $N=N(\varepsilon, M)=\left[M \varepsilon^{-\alpha p /(\alpha-p)}\right]$, where [.] denotes the integer part.

Now, since $\Psi$ is continuous, Assumption (A.1) implies that

$$
\Delta_{n}=\sup _{x}\left|P\left(\left|Z_{n}\right| \geq n^{1 / \alpha} x\right)-\Psi(x)\right| \rightarrow 0 \quad \text { as } \quad n \rightarrow \infty,
$$

hence also (cf., e.g., Gut [8], Lemma A.6.1) that

$$
\lim _{N \rightarrow \infty} N^{1-r / p} \sum_{n \leq N} n^{r / p-2} \Delta_{n}=0 .
$$

Since $N=N(\varepsilon, M) \rightarrow \infty$ as $\varepsilon \searrow 0$, this results in

$$
\begin{gathered}
\varepsilon^{(\alpha p /(\alpha-p))(r / p-1)} \sum_{n \leq N} n^{r / p-2}\left|P\left(\left|Z_{n}\right| \geq \varepsilon n^{1 / p}\right)-\Psi\left(\varepsilon n^{1 / p-1 / \alpha}\right)\right| \\
\leq \varepsilon^{(\alpha p /(\alpha-p))(r / p-1)} N^{r / p-1} N^{1-r / p} \sum_{n \leq N} n^{r / p-2} \Delta_{n} \\
\leq M^{r / p-1} N^{1-r / p} \sum_{n \leq N} n^{r / p-2} \Delta_{n} \rightarrow 0 \text { as } \varepsilon \searrow 0 .
\end{gathered}
$$

Next we note that, in view of Assumption (A.2), with $x=\varepsilon n^{1 / p-1 / \alpha}$,

$$
\left|P\left(\left|Z_{n}\right| \geq \varepsilon n^{1 / p}\right)-P\left(|Z| \geq \varepsilon n^{1 / p-1 / \alpha}\right)\right| \leq C \varepsilon^{-r} n^{-r / p+r / \alpha}
$$


for all $n$, so that, since $r / \alpha<1$,

$$
\begin{aligned}
\sum_{n>N} & n^{r / p-2}\left|P\left(\left|Z_{n}\right| \geq \varepsilon n^{1 / p}\right)-\Psi\left(\varepsilon n^{1 / p-1 / \alpha}\right)\right| \leq C \varepsilon^{-r} \sum_{n>N} n^{r / \alpha-2} \\
& \leq C \varepsilon^{-r} N^{r / \alpha-1} \leq C M^{r / \alpha-1} \varepsilon^{-r}\left(\varepsilon^{-\alpha p /(\alpha-p)}\right)^{r / \alpha-1} \\
& =C M^{r / \alpha-1} \varepsilon^{-\alpha(r-p) /(\alpha-p)}
\end{aligned}
$$

Therefore,

$$
\limsup _{\varepsilon \searrow 0} \varepsilon^{(\alpha p /(\alpha-p))(r / p-1)} \sum_{n>N} n^{r / p-2}\left|P\left(\left|Z_{n}\right| \geq \varepsilon n^{1 / p}\right)-\Psi\left(\varepsilon n^{1 / p-1 / \alpha}\right)\right| \leq C M^{r / \alpha-1} .
$$

On combining (2.6) and (2.7) and letting $M \rightarrow \infty$, the proof of (2.5) is complete.

Proof of Theorem 2.1. Combine Propositions 2.1 and 2.2.

REMARK 2.3 The arguments in the proof of (2.7) above show, in particular, that, under Assumption (A.2), we have, for all $0<p<r(<\alpha)$,

$$
\sum_{n=1}^{\infty} n^{r / p-2} P\left(\left|Z_{n}\right| \geq \varepsilon n^{1 / p}\right)<\infty \quad \text { for all } \varepsilon>0
$$

(cf. Theorem 1.2).

Under a somewhat stronger assumption, we also have an extension of Theorem 2.1 to the case of $\alpha=2$ and $r \geq 2$.

(A.3) For some $r \geq 2$ there are constants $C>0$ and $q \geq 2$ such that, for all $n \geq 1$ and $x>0$,

$$
\max \left\{P(|Z| \geq x), P\left(\left|Z_{n}\right| / n^{1 / 2} \geq x\right)\right\} \leq C\left\{n Q_{1}\left(n^{1 / 2} x\right)+Q_{2}(x)\right\},
$$

where $Q_{1}$ and $Q_{2}$ are non-increasing functions such that

$$
\int_{1}^{\infty} x^{r-1} Q_{1}(x) d x<\infty \text { and } \int_{1}^{\infty} x^{q-1} Q_{2}(y) d y<\infty .
$$

Note for later use that the conditions on $Q_{1}$ and $Q_{2}$ above imply that $\max \left\{E|Z|^{r}, E|Z|^{q}\right\}<\infty$.

Theorem 2.2 Under Assumptions (A.1) (with $\alpha=2)$ and (A.3) (with $q=2(r-p) /(2-p)$ ), we have, for $0<p<2$,

$$
\lim _{\varepsilon \searrow 0} \varepsilon^{2(r-p) /(2-p)} \sum_{n=1}^{\infty} n^{r / p-2} P\left(\left|Z_{n}\right| \geq \varepsilon n^{1 / p}\right)=\frac{p}{r-p} E|Z|^{2(r-p) /(2-p)} .
$$

The proof of Theorem 2.2 is just a slight modification of the proof of Theorem 2.1 and will be based on the following two propositions.

Proposition 2.3 Under the assumptions of Theorem 2.2, we have, for $0<p<2$,

$$
\lim _{\varepsilon \searrow 0} \varepsilon^{2(r-p) /(2-p)} \sum_{n=1}^{\infty} n^{r / p-2} P\left(|Z| \geq \varepsilon n^{1 / p-1 / 2}\right)=\frac{p}{r-p} E|Z|^{2(r-p) /(2-p)} .
$$

Proof. We first assume that $r / p \leq 2$, i.e., that $p \geq r / 2$. Then, with $\Psi(x)=P(|Z| \geq x)$,

$$
\int_{1}^{\infty} x^{r / p-2} \Psi\left(\varepsilon x^{1 / p-1 / 2}\right) d x \leq \sum_{n=1}^{\infty} n^{r / p-2} \Psi\left(\varepsilon n^{1 / p-1 / 2}\right) \leq \int_{0}^{\infty} x^{r / p-2} \Psi\left(\varepsilon x^{1 / p-1 / 2}\right) d x
$$


By the change of variable $y=\varepsilon x^{1 / p-1 / 2},(2.10)$ yields

$$
\begin{aligned}
\frac{2 p}{2-p} \int_{\varepsilon}^{\infty} y^{2(r-p) /(2-p)-1} \Psi(y) d y & \leq \varepsilon^{2(r-p) /(2-p)} \sum_{n=1}^{\infty} n^{r / p-2} \Psi\left(\varepsilon n^{1 / p-1 / 2}\right) \\
& \leq \frac{2 p}{2-p} \int_{0}^{\infty} y^{2(r-p) /(2-p)-1} \Psi(y) d y
\end{aligned}
$$

and since

$$
\int_{0}^{\infty} y^{2(r-p) /(2-p)-1} \Psi(y) d y=\frac{2-p}{2(r-p)} E|Z|^{2(r-p) /(2-p)},
$$

(2.9) is immediate from (2.11).

For the case $r / p>2$, i.e., $0<p<r / 2$, we just have to make the necessary modifications similar to the proof of Proposition 2.1. Details are omitted.

Proposition 2.4 Under the assumptions of Theorem 2.2, we have, for $0<p<2$,

$$
\lim _{\varepsilon \searrow 0} \varepsilon^{2(r-p) /(2-p)} \sum_{n=1}^{\infty} n^{r / p-2}\left|P\left(\left|Z_{n}\right| \geq \varepsilon n^{1 / p}\right)-P\left(|Z| \geq \varepsilon n^{1 / p-1 / 2}\right)\right|=0 .
$$

Proof. Set, for $0<\varepsilon \leq 1$ and $M \geq 1, N=N(\varepsilon, M)=\left[M \varepsilon^{-2 p /(2-p)}\right]$.

Since $\Psi$ is continuous, Assumption (A.1) implies that

$$
\Delta_{n}=\sup _{x}\left|P\left(\left|Z_{n}\right| \geq n^{1 / \alpha} x\right)-\Psi(x)\right| \rightarrow 0 \quad \text { as } \quad n \rightarrow \infty .
$$

Since $N=N(\varepsilon, M) \rightarrow \infty$ as $\varepsilon \searrow 0$, we therefore obtain, along the lines of the proof of Proposition 2.2 , that

$$
\begin{gathered}
\varepsilon^{2(r-p) /(2-p)} \sum_{n \leq N} n^{r / p-2}\left|P\left(\left|Z_{n}\right| \geq \varepsilon n^{1 / p}\right)-\Psi\left(\varepsilon n^{1 / p-1 / 2}\right)\right| \\
\leq M^{r / p-1} N^{1-r / p} \sum_{n \leq N} n^{r / p-2} \Delta_{n} \rightarrow 0 \quad \text { as } \varepsilon \searrow 0 .
\end{gathered}
$$

In view of Assumption (A.3), with $x=\varepsilon n^{1 / p-1 / 2}$, we have

$$
\begin{aligned}
& \sum_{n>N} n^{r / p-2}\left|P\left(\left|Z_{n}\right| \geq \varepsilon n^{1 / p}\right)-\Psi\left(\varepsilon n^{1 / p-1 / 2}\right)\right| \\
& \quad \leq C\left\{\int_{N+1}^{\infty} x^{r / p-1} Q_{1}\left(\varepsilon x^{1 / p}\right) d x+\int_{N+1}^{\infty} x^{r / p-2} Q_{2}\left(\varepsilon x^{1 / p-1 / 2}\right) d x\right\}=I_{N 1}+I_{N 2} .
\end{aligned}
$$

Note that $N+1>M \varepsilon^{-2 p /(2-p)}$. By the change of variable $y=\varepsilon x^{1 / p}$, we first have

$$
\varepsilon^{2(r-p) /(2-p)} I_{N 1} \leq C \varepsilon^{p(r-2)((2-p)} \int_{M^{1 / p} \varepsilon^{-p /(2-p)}}^{\infty} y^{r-1} Q_{1}(y) d y,
$$

whence

$$
\lim _{M \rightarrow \infty} \limsup _{\varepsilon \searrow 0} \varepsilon^{2(r-p) /(2-p)} I_{N 1}=0,
$$

thanks to Assumption (2.3), and the fact that $r \geq 2$ and $0<p<2$.

Next, by the change of variable $y=\varepsilon x^{1 / p-1 / 2}$, we get for the second integral that

$$
\varepsilon^{2(r-p) /(2-p)} I_{N 2} \leq C \int_{M^{(2-p) /(2 p)}}^{\infty} y^{2(r-p) /(2-p)-1} Q_{2}(y) d y .
$$

An application of Assumption (2.3) with $q=2(r-p) /(2-p)$, therefore also tells us that

$$
\lim _{M \rightarrow \infty} \limsup _{\varepsilon \searrow 0} \varepsilon^{2(r-p) /(2-p)} I_{N 2}=0 .
$$

A final combination of $(2.13)-(2.15)$ completes the proof of $(2.12)$.

Proof of Theorem 2.2. Combine Propositions 2.3 and 2.4. 
REMARK 2.4 The arguments in the proof of (2.14) and (2.15) show, in particular, that, under Assumption (A.3), for $0<p<2$ and $r \geq 2$,

$$
\sum_{n=1}^{\infty} n^{r / p-2} P\left(\left|Z_{n}\right| \geq \varepsilon n^{1 / p}\right)<\infty \quad \text { for all } \varepsilon>0
$$

(cf. Theorem 1.2 with $r \geq 2$ ).

Next we turn our attention to the limiting case $r=p$, which is not covered by Theorems 2.1 and 2.2 (cf. Gut and Spătaru [9], Theorem 2, for case of sums of i.i.d. random variables).

Theorem 2.3 Let $0<r<\alpha$. Under Assumptions (A.1) and (A.2) we have

$$
\lim _{\varepsilon \searrow 0} \frac{1}{-\log \varepsilon} \sum_{n=1}^{\infty} \frac{1}{n} P\left(\left|Z_{n}\right| \geq \varepsilon n^{1 / r}\right)=\frac{\alpha r}{\alpha-r} .
$$

Similarly as above, the proof of Theorem 2.3 is based on the following two propositions.

Proposition 2.5 Let $0<r<\alpha$. Under Assumption (A.1) we have

$$
\lim _{\varepsilon \searrow 0} \frac{1}{-\log \varepsilon} \sum_{n=1}^{\infty} \frac{1}{n} P\left(|Z| \geq \varepsilon n^{1 / r-1 / \alpha}\right)=\frac{\alpha r}{\alpha-r} .
$$

Proof. The proof is a slight modification of the proof of Proposition 2.1. We have

$$
\int_{2}^{\infty} \frac{1}{x} \Psi\left(\varepsilon x^{1 / r-1 / \alpha}\right) d x \leq \sum_{n=1}^{\infty} \frac{1}{n} \Psi\left(\varepsilon n^{1 / r-1 / \alpha}\right) \leq 1+\int_{1}^{\infty} \frac{1}{x} \Psi\left(\varepsilon x^{1 / r-1 / \alpha}\right) d x .
$$

By the change of variable $y=\varepsilon x^{1 / r-1 / \alpha},(2.18)$ yields

$$
\frac{\alpha r}{\alpha-r} \int_{\varepsilon C}^{\infty} \frac{1}{y} \Psi(y) d y \leq \sum_{n=1}^{\infty} \frac{1}{n} \Psi\left(\varepsilon n^{1 / r-1 / \alpha}\right) \leq \frac{\alpha r}{\alpha-r} \int_{\varepsilon}^{\infty} \frac{1}{y} \Psi(y) d y .
$$

Since, in view of $\lim _{y \searrow 0} \Psi(y)=1$, for all $C>0$,

$$
\lim _{\varepsilon \searrow 0} \frac{1}{-\log \varepsilon} \int_{\varepsilon C}^{\infty} \frac{1}{y} \Psi(y) d y=1,
$$

(2.17) follows immediately from (2.19), which completes the proof.

Proposition 2.6 Let $0<r<\alpha$. Under Assumptions (A.1) and (A.2) we have

$$
\lim _{\varepsilon \searrow 0} \frac{1}{-\log \varepsilon} \sum_{n=1}^{\infty} \frac{1}{n}\left|P\left(\left|Z_{n}\right| \geq \varepsilon n^{1 / r}\right)-P\left(|Z| \geq \varepsilon n^{1 / r-1 / \alpha}\right)\right|=0 .
$$

Proof. For $0<\varepsilon \leq 1$ set $N=N(\varepsilon)=\left[\varepsilon^{-\gamma}\right]$, with $\gamma>0$ to be specified below.

Since, by Assumption (A.1),

$$
\Delta_{n}=\sup _{x}\left|P\left(\left|Z_{n}\right| \geq n^{1 / \alpha} x\right)-\Psi(x)\right| \rightarrow 0 \quad \text { as } \quad n \rightarrow \infty,
$$

we also have

$$
\lim _{N \rightarrow \infty} \frac{1}{\log N} \sum_{n \leq N} \frac{1}{n} \Delta_{n}=0 .
$$

Since $N=N(\varepsilon) \rightarrow \infty$ as $\varepsilon \searrow 0$, this tells us that

$$
\begin{aligned}
& \frac{1}{-\log \varepsilon} \sum_{n \leq N} \frac{1}{n}\left|P\left(\left|Z_{n}\right| \geq \varepsilon n^{1 / r}\right)-\Psi\left(\varepsilon n^{1 / r-1 / \alpha}\right)\right| \\
& \quad \leq \frac{1}{-\log \varepsilon} \log N \frac{1}{\log N} \sum_{n \leq N} \frac{1}{n} \Delta_{n} \leq C \frac{1}{\log N} \sum_{n \leq N} \frac{1}{n} \Delta_{n} \rightarrow 0 \quad \text { as } \quad \varepsilon \searrow 0 .
\end{aligned}
$$


An appeal to Assumption (A.2) with $x=\varepsilon n^{1 / r-1 / \alpha}$ now yields

$$
\left|P\left(\left|Z_{n}\right| \geq \varepsilon n^{1 / r}\right)-P\left(|Z| \geq \varepsilon n^{1 / r-1 / \alpha}\right)\right| \leq C \varepsilon^{-r} n^{-1+r / \alpha}
$$

for all $n$, so that, since $r / \alpha<1$,

$$
\begin{gathered}
\sum_{n>N} \frac{1}{n}\left|P\left(\left|Z_{n}\right| \geq \varepsilon n^{1 / p}\right)-\Psi\left(\varepsilon n^{1 / r-1 / \alpha}\right)\right| \leq C \varepsilon^{-r} \sum_{n>N} n^{r / \alpha-2} \\
\leq C \varepsilon^{-r} N^{r / \alpha-1} \leq C \varepsilon^{-r} \varepsilon^{\gamma(\alpha-r) / \alpha} .
\end{gathered}
$$

Therefore, if $-r+\gamma(\alpha-r) / \alpha>0$, i.e., $\gamma>\alpha r /(\alpha-r)$, we obtain

$$
\lim _{\varepsilon \searrow 0} \frac{1}{-\log \varepsilon} \sum_{n>N} \frac{1}{n}\left|P\left(\left|Z_{n}\right| \geq \varepsilon n^{1 / p}\right)-\Psi\left(\varepsilon n^{1 / p-1 / \alpha}\right)\right|=0,
$$

so that a combination of (2.21) and (2.22) completes the proof of (2.20).

For the limiting case $p=r=2$ in Theorem 2.2 we obtain the following result (cf. Gut and Spătaru [9], Theorem 3, in case of sums of i.i.d. random variables).

Theorem 2.4 Under Assumptions (A.1) (with $\alpha=2$ ) and (A.3) (with $r=2$ and $q=2 \delta+2$ ), we have, for $0 \leq \delta \leq 1$,

$$
\lim _{\varepsilon \searrow 0} \varepsilon^{2 \delta+2} \sum_{n=1}^{\infty} \frac{(\log n)^{\delta}}{n} P\left(\left|Z_{n}\right| \geq \varepsilon \sqrt{n \log n}\right)=\frac{1}{\delta+1} E|Z|^{2 \delta+2} .
$$

The proof of Theorem 2.4 is again a modification of the proof of Theorem 2.1 and will be based on the following two propositions.

Proposition 2.7 Under the assumptions of Theorem 2.4 we have

$$
\lim _{\varepsilon \searrow 0} \varepsilon^{2 \delta+2} \sum_{n=1}^{\infty} \frac{(\log n)^{\delta}}{n} P(|Z| \geq \varepsilon \sqrt{\log n})=\frac{1}{\delta+1} E|Z|^{2 \delta+2} .
$$

Proof. We have, with $\Psi(x)=P(|Z| \geq x)$,

$$
\int_{3}^{\infty} \frac{(\log x)^{\delta}}{x} \Psi(\varepsilon \sqrt{\log x}) d x \leq \sum_{n=3}^{\infty} \frac{(\log n)^{\delta}}{n} \Psi(\varepsilon \sqrt{\log n}) \leq \int_{2}^{\infty} \frac{(\log x)^{\delta}}{x} \Psi(\varepsilon \sqrt{\log x}) d x .
$$

By the change of variable $y=\varepsilon \sqrt{\log x},(2.25)$ yields

$$
\int_{\varepsilon \sqrt{\log 3}}^{\infty} 2 y^{2 \delta+1} \Psi(y) d y \leq \varepsilon^{2 \delta+2} \sum_{n=3}^{\infty} \frac{(\log n)^{\delta}}{n} \Psi(\varepsilon \sqrt{\log n}) \leq \int_{\varepsilon \sqrt{\log 2}}^{\infty} 2 y^{2 \delta+1} \Psi(y) d y .
$$

Since

$$
\int_{0}^{\infty} 2 y^{2 \delta+1} \Psi(y) d y=\frac{1}{\delta+1} E|Z|^{2 \delta+2}
$$

(2.24) is immediate from (2.26).

Proposition 2.8 Under the assumptions of Theorem 2.4 we have

$$
\lim _{\varepsilon \searrow 0} \varepsilon^{2 \delta+2} \sum_{n=1}^{\infty} \frac{(\log n)^{\delta}}{n}\left|P\left(\left|Z_{n}\right| \geq \varepsilon \sqrt{n \log n}\right)-P(|Z| \geq \varepsilon \sqrt{\log n})\right|=0 .
$$


Proof. Let $0<\varepsilon \leq 1$ and $M \geq 1$, and set $N=N(\varepsilon, M)=\left[\exp \left(M / \varepsilon^{2}\right)\right]$.

Again, Assumption (A.1) implies that

$$
\Delta_{n}=\sup _{x}\left|P\left(\left|Z_{n}\right| \geq \sqrt{n} x\right)-\Psi(x)\right| \rightarrow 0 \quad \text { as } \quad n \rightarrow \infty
$$

so that, by arguing as in the proof of Proposition 2.2, recalling that $N=N(\varepsilon, M) \rightarrow \infty$ as $\varepsilon \searrow 0$,

$$
\begin{aligned}
\varepsilon^{2 \delta+2} & \sum_{n \leq N} \frac{(\log n)^{\delta}}{n}\left|P\left(\left|Z_{n}\right| \geq \varepsilon \sqrt{n \log n}\right)-\Psi(\varepsilon \sqrt{\log n})\right| \\
& \leq C M^{\delta+1}(\log N)^{-(\delta+1)} \sum_{n \leq N} \frac{(\log n)^{\delta}}{n} \Delta_{n} \rightarrow 0 \quad \text { as } \quad \varepsilon \searrow 0 .
\end{aligned}
$$

In view of Assumption (A.3), with $x=\varepsilon \sqrt{\log n}$, we have

$$
\begin{aligned}
& \sum_{n>N} \frac{(\log n)^{\delta}}{n}\left|P\left(\left|Z_{n}\right| \geq \varepsilon \sqrt{n \log n}\right)-\Psi(\varepsilon \sqrt{\log n})\right| \\
& \quad \leq C\left\{\int_{N+1}^{\infty}(\log x)^{\delta} Q_{1}\left(\sqrt{\varepsilon^{2} x \log x}\right) d x+\int_{N+1}^{\infty} \frac{(\log x)^{\delta}}{x} Q_{2}\left(\sqrt{\varepsilon^{2} \log x}\right) d x\right\}=I_{N 1}+I_{N 2} .
\end{aligned}
$$

Note that $N+1>\exp \left(M / \varepsilon^{2}\right)$. Since $0 \leq \delta \leq 1$, a change of variable $y=\varepsilon^{2} x \log x$ and $y^{\prime} \sim$ $\varepsilon^{2} \log x \sim \varepsilon^{2} \log y$ as $x \rightarrow \infty$ in the first integral, yields

$$
\begin{aligned}
\varepsilon^{2 \delta+2} I_{N 1} & \leq C \varepsilon^{2 \delta+2} \int_{M \exp \left(M / \varepsilon^{2}\right)}^{\infty} \varepsilon^{-2}(\log y)^{\delta-1} Q_{1}(\sqrt{y}) d y \\
& \leq C \int_{M \exp \left(M / \varepsilon^{2}\right)}^{\infty} Q_{1}(\sqrt{y}) d y=2 C \int_{\sqrt{M \exp \left(M / \varepsilon^{2}\right)}}^{\infty} z Q_{1}(z) d z .
\end{aligned}
$$

Hence,

$$
\limsup _{\varepsilon \searrow 0} \varepsilon^{2 \delta+2} I_{N 1}=0
$$

due to Assumption (A.3) (with $r=2$ ), for all $M \geq 1$.

Next, by the change of variable $y=\varepsilon^{2} \log x$, we get for the second integral that

$$
\varepsilon^{2 \delta+2} I_{N 2} \leq C \int_{M}^{\infty} y^{\delta} Q_{2}(\sqrt{y}) d y=2 C \int_{\sqrt{M}}^{\infty} z^{2 \delta+1} Q_{2}(z) d z .
$$

Therefore, in view of Assumption (2.3) (with $q=2 \delta+2$ ), also

$$
\lim _{M \rightarrow \infty} \limsup _{\varepsilon \searrow 0} \varepsilon^{2 \delta+2} I_{N 2}=0,
$$

so that a combination of $(2.28)-(2.30)$ completes the proof of $(2.27)$.

Proof of Theorem 2.4. Combine Propositions 2.7 and 2.8.

REMARK 2.5 The arguments in the proof of (2.29) and (2.30) show, in particular, that, under Assumption (A.3) (with $r=2$ and $q=2 \delta+2$, for $0 \leq \delta \leq 1$ ),

$$
\sum_{n=1}^{\infty} \frac{(\log n)^{\delta}}{n} P\left(\left|Z_{n}\right| \geq \varepsilon \sqrt{n \log n}\right)<\infty \quad \text { for all } \varepsilon>0 .
$$

\section{$3 \quad$ Examples}

The aim of this section is to demonstrate, by a series of examples, that the general pattern of the proofs, which is essentially adapted from the i.i.d. case, is also applicable under much weaker conditions as well as for other models. For the sake of completeness, however, we begin by briefly mentioning, in Example 3.1, some precise asymptotics for partial sums of i.i.d. summands, since, after all, everything started from there. 


\subsection{Examples in terms of weaker assumptions}

In this subsection we mention some examples of results on precise asymptotics under weaker (than i.i.d.) assumptions. Following is a list of assumptions that we have encountered, either in the literature or as referees of manuscripts, some of which have been published and some not. We therefore give no complete list of references, but, rather, invite the readers to search on the web for any model of interest.

1. Partial sums of i.i.d. random variables;

2. Attraction to semistable laws;

3. Independence, but not i.i.d. under e.g. domination assumptions, such as

$$
\left\{\begin{array}{l}
\left|X_{n}\right| \leq|Y|, \quad \text { or } \\
P\left(\left|X_{n}\right|>x\right) \leq P(|Y|>x), \quad \text { or } \\
\frac{1}{n} \sum_{k=1}^{n} P\left(\left|X_{k}\right|>x\right) \leq P(|Y|>x)
\end{array}\right.
$$

for all $n$ and some random variable $Y$ with mean 0 ;

4. Strict stationarity under various mixing assumptions;

5. Negative association;

6. Positive association;

7. Association;

8. Weighted sums $-\sum_{n=1}^{\infty} n^{(r / p)-2} P\left(\left|\sum_{k=1}^{n} a_{k} X_{k}\right|>\varepsilon b_{n}\right)$;

9. More general weights $-\sum_{n=1}^{\infty} h(n) P\left(\left|S_{n}\right|>\varepsilon b_{n}\right)$;

10. Power sums;

11. Moving average;

12. Linear processes;

13. Linearly positively quadrant dependent;

14. Martingale difference sequences.

Since our results concern rates, we assume in our discussion that Condition (A.1) is "automatically" satisfied; after all, if there is no convergence in the outset, any discussion about rates is void.

EXAMPLE 3.1 (The i.i.d. case)

Spătaru [28], followed by Gut and Spătaru [9], obtained versions of Theorems 2.1 and 2.3-2.4, respectively, in the case of partial sums $S_{n}$ of i.i.d. random variables belonging to the (normal) domain of attraction of a stable law with index $\alpha \in(1,2]$ (see, e.g., Theorems 1.3-1.5 above). Here, Steps I and IIa can easily be verified via properties of the limiting (stable or normal) random variable (say) $Z$, and Step IIb can be taken care of by tail estimates for $Z$ in combination with an application of the Fuk-Nagaev [7] inequality for $S_{n}$ (see Spătaru [28], Lemmas 1 and 2, and Gut and Spătaru [9], Lemmas 2.1-2.3).

EXAMPLE 3.2 (Attraction to semistable laws)

Scheffler [24] extends Theorem 1.5 to partial sums $S_{n}$ of i.i.d. random variables belonging to the domain of attraction of a semistable law with index $\alpha \in(0,2)$. Here, again, Steps I and IIa are verified via properties of the limiting semistable random variable ([24], Lemmas 3.1, 3.2, and 3.4), and Step IIb can then be taken care of by an appropriate large deviation estimate for $S_{n}$, e.g., Heyde [14] (see [24], Lemma 4.4).

Using a similar approach, Scheffler [24] also presents a result for the limiting case $p=\alpha$, but under the stronger assumption that the summands belong to the normal domain of attraction of a semistable or even a stable law. 
EXAMPLE 3.3 (Domination)

Precise asymptotics also extend to independent, not necessarily i.i.d. sequences under domination assumptions, since domination of summands carries over to sums, so that any tail- or moment estimate for the original sequence is automatic from the corresponding one for an i.i.d. sequence.

EXAMPle 3.4 (Positive association)

Mi [22] studied precise asymptotics for the partial sums $\left\{S_{n}, n \geq 1\right\}$ of a sequence $\left\{X_{i}, i \geq 1\right\}$ of strictly stationary, positively associated random variables with mean zero and finite $(2+\delta)$-th moment. Let $\sigma^{2}=E X_{1}^{2}+2 \sum_{j=2}^{\infty} E X_{1} X_{j}>0$. Then, under the assumptions given there, Mi's Theorems 1.1 and 1.2, for example, are special cases of our Theorems 2.1 and 2.3 above, with $\alpha=2, Z_{n}=S_{n}$, and $Z$ being an $N\left(0, \sigma^{2}\right)$-random variable. They can even be extended to cover the case $0<p<1$.

Concerning our assumptions, we note that (A.1) follows from Newman's [23] central limit theorem for associated sequences. This takes care of Steps I and IIa. Moreover, since the tails of a normal distribution function are exponentially bounded, Assumption (A.2), and thus Step IIb, can be obtained via Markov's inequality, since, for some $2<t<2+\delta$ and $C>0$, one has $E\left|S_{n}\right|^{t} \leq C n^{t / 2}$ (cf. Mi [22], p. 200).

Note that our Assumption (A.3) can be verified in a similar way by an application of Markov's inequality, which implies that Theorems 2.2 and 2.4 are also applicable to positively associated sequences.

EXAMPLE 3.5 (Weighted sums)

Cheng and Wang [4] studied precise asymptotics for sums $\sum_{n=1}^{\infty} h^{\prime}(n) P\left(\left|S_{n}-a_{n}\right|>\varepsilon b_{n} g(h(n))\right)$, when the limit suitably normalized equals $E|Z| \varrho$. The main assumption is that the distribution function of the summands belongs to the domain of attraction of a nondegenerate stable law $G_{\alpha}$, and $Z \in G_{\alpha}$, with $0<\varrho<\alpha \leq 2$. Furthermore, there are various assumptions on $h$ and $g$.

The crucial step here corresponds to Step IIb in our discussion, and is taken care of in their Proposition 4 via a uniform bound on $E\left|\left(S_{n}-a_{n}\right) / b_{n}\right|^{q}$, where $\varrho<q<\alpha$, and Markov's inequality.

EXAMPLE 3.6 (Negative association)

Huang and Zhang [17] consider the case of negatively associated summands. Step IIb is here taken care of as in, say, [9] with the Fuk-Nagaev [7] inequality being replaced by an analog due to Shao $[27]$.

Another reference along these lines is [3].

\section{EXAMPLE 3.7 (@-mixing)}

Huang, Jiang, and Zhang [18] consider the case of $\varrho$-mixing summands and have a layout similar to that of [17]. In addition to treating sums, they also consider maximal partial sums. In their first result, Step IIb is dealt with in their Proposition 3.4. For the case $1 \leq p<r<2$, the approach is reminiscent to that of [4]. The case $r>2$ exploits a maximal inequality due to Shao [25] (in the spirit of Fuk-Nagaev [7], although more involved) followed by "the usual estimates", which, however, are technically rather intricate. One easily observes (again) that Step IIb is the only part of the proof that requires work (which clearly illustrates the main theme of the present paper, without any attempt to criticize).

In their second result they exploit the first proof, and in the third result, the hard part is the analogous Proposition 5.4, where another Fuk-Nagaev inequality for $\varrho$-mixing summands ([26]) is exploited.

\section{EXAMPLE 3.8 (Linear processes)}

Tan and Yang [30] study linear processes, that is, sums in which the summands have the form $X_{k}=\sum_{j=1}^{\infty} a_{j} \varepsilon_{k-j}, k \geq 1$, and $\left\{\varepsilon_{k}, k \geq 1\right\}$ is a stationary sequence of (positively) associated random variables with mean zero. They prove two theorems for weighted sums of the kind referred to in Example 3.5. The crucial steps are uniform bounds of the Marcinkiewicz-Zygmund type for the normalized moments of the partial sums of the epsilons, which is exploited in their Propositions 3.4 and 4.4 .

EXAMPLE 3.9 (Martingale differences)

Haeusler [13] derived convergence rates in the functional central limit theorem for certain martingale difference sequences satisfying, among other things, a $(2+\delta)$-th moment condition (cf., e.g., 
[13], Theorem 2). Naturally, this immediately implies a central limit theorem, so that Assumption (A.1), and thus Steps I and IIa are immediate for such sequences.

Moreover, a key ingredient in the proofs is a Fuk-Nagaev type inequality ([13], Lemma 1), which, if e.g. the martingale differences are identically distributed, can easily be used to verify our Assumption (A.3), and thus take care of Step IIb as well. This shows that precise asymptotics are also available for martingale differences along the lines of Theorems 2.2 and 2.4, respectively.

\subsection{Other settings}

Just as there exist laws of large numbers or central limit theorems for sums, there exist parallel results for other specific models. Following the general pattern of proofs, discussed in Section 2.1, precise asymptotics are available for such settings as well, e.g., for

1. Maximal sums: $\max _{1 \leq k \leq n}\left|S_{k}\right|$ instead of $\left|S_{n}\right|$;

2. Moments: $\sum_{n=1}^{\infty} \frac{1}{n^{p}} E\left|S_{n}\right|^{p} I\left\{\left|S_{n}\right|>\varepsilon n\right\}, 0<p<2$ instead of $\left|S_{n}\right|$;

3. Centered max-moments: $E\left|\max _{1 \leq k \leq n} S_{k}-\varepsilon n^{\sigma}\right|$ instead of $S_{n}$;

4. Self-normalized sums;

5. Results related to the law of the iterated logarithm;

6. $R / S$-statistics;

7. Order statistics;

8. Extremes;

9. Renewal processes and the associated counting process;

10. Records and record times;

11. Products;

12. Empirical processes;

13. Hilbert space valued random elements;

14. Banach space valued random elements;

15. Random fields;

16. You name it.

\section{Rates}

With every limit theorem there is a rate result associated with it. As for the law of large numbers we have the Baum-Katz theorem, Theorem 1.2 above. As for the central limit theorem we have the classical Berry-Esseen theorem.

In the present context Klesov [20] proved the following convergence rate result related to Heyde's [15] result (1.2).

Theorem 4.1 Let $X, X_{1}, X_{2}, \ldots$ be i.i.d. random variables, and set $S_{n}=\sum_{k=1}^{n} X_{k}, n \geq 1$.

(a) If $X$ is normal with mean 0 and variance $\sigma^{2}>0$, then

$$
\lim _{\varepsilon \searrow 0}\left(\sum_{n=1}^{\infty} P\left(\left|S_{n}\right| \geq \varepsilon n\right)-\frac{\sigma^{2}}{\varepsilon^{2}}\right)=-\frac{1}{2} .
$$

(b) If $E X=0, E X^{2}=\sigma^{2}>0$, and $E|X|^{3}<\infty$, then

$$
\lim _{\varepsilon \searrow 0} \varepsilon^{3 / 2}\left(\sum_{n=1}^{\infty} P\left(\left|S_{n}\right| \geq \varepsilon n\right)-\frac{\sigma^{2}}{\varepsilon^{2}}\right)=0 .
$$


Klesov's [20] result was recently extended to provide rate results for all of Theorems $1.3-1.5$ by the authors in $[11,12]$, to which we refer for exact formulations.

Our final remark is that, so far, to the best of our knowledge, rate results exist only in the i.i.d. case. It is also an open question whether or not there exists a (more) general rate analog to our results.

\section{References}

[1] Baum, L.E., And Katz, M. (1965). Convergence rates in the law of large numbers. Trans. Amer. Math. Soc. 120, 108-123.

[2] Chen, R. (1978). A remark on the tail probability of a distribution. J. Multivariate Analysis 8, 328-333.

[3] Cheng, F.-Y. And Wang, Y.-B. (2004). Precise asymptotics of partial sums for iid and NA sequences. (In Chinese.) Acta Math. Sinica (Chinese Series) 47, 965-972.

[4] Cheng, F.-Y. And Wang, Y.-B. (2006). A remark on the precise asymptotics in the BaumKatz laws of large numbers. Can. Appl. Math. Q. 14, 33-41.

[5] Erdős, P. (1949). On a theorem of Hsu and Robbins. Ann. Math. Statist. 20, 286-291.

[6] Erdős, P. (1950). Remark on my paper "On a theorem of Hsu and Robbins". Ann. Math. Statist. 21, 138.

[7] Fuk, D.H., And Nagaev, S.V. (1971). Probability inequalities for sums of independent random variables. Theor. Probab. Appl. 16, 643-660.

[8] Gut, A. (2007). Probability: A Graduate Course, Springer-Verlag, New York, Corr. 2nd printing.

[9] Gut, A., And Spătaru, A. (2000a). Precise asymptotics in the Baum-Katz and Davis law of large numbers. J. Math. Anal. Appl. 248, 233-246.

[10] Gut, A., and Spătaru, A. (2000b). Precise asymptotics in the law of the iterated logarithm. Ann. Probab. 28, 1870-1883.

[11] Gut, A., And Steinebach J. (2011a). Convergence rates in precise asymptotics. U.U.D.M. Report 2011:11 (submitted).

[12] Gut, A., And Steinebach J. (2011b). Convergence rates in precise asymptotics II. U.U.D.M. Report 2011:15 (submitted).

[13] Haeusler, E. (1984). An exact rate of convergence in the functional central limit theorem for special martingale difference arrays. Z. Wahrsch. Verw. Geb. 65, 523-534.

[14] Heyde, C.C. (1967). On large deviation problems for sums of random variables not attracted to the normal law. Ann. Math. Statist. 38, 1575-1578.

[15] Heyde, C.C. (1975). A supplement to the strong law of large numbers. J. Appl. Probab. 12, 173-175.

[16] Hsu, P.L. And Robbins, H. (1947). Complete convergence and the law of large numbers. Proc. Nat. Acad. Sci. USA 33, 25-31.

[17] Huang, W. And Zhang, L.-X. Precise asymptotics in the Baum-Katz and Davis laws of large numbers for NA sequences. Preprint.

[18] Huang, W., Jiang, Y., And Zhang, L.-X. (2005). Precise asymptotics in the Baum-Katz and Davis laws of large numbers for $\varrho$-mixing sequences. Acta Math. Sinica 21, 1057-1070.

[19] Katz, M. (1963). The probability in the tail of a distribution. Ann. Math. Statist. 34, 312-318. 
[20] Klesov, O.I. (1994). On the convergence rate in a theorem of Heyde. Theory Probab. Math. Statist. 49, 83-87 (1995); translated from Teor. Imovīr. Mat. Stat. 49 (1993), 119125 (Ukrainian).

[21] Marcinkiewicz, J., And Zygmund, A. (1937). Sur les fonctions indépendantes. Fund. Math. 29, 60-90.

[22] Mi, C. (2005). Precise asymptotics in the Baum-Katz and Davis law of large numbers for positively associated sequences. Appl. Math. J. Chinese Univ., Ser. B, 20(2), 197-204.

[23] Newman, C.M. (1980). Normal fluctuations and the FKG inequalities. Comm. Math. Phys. 74, 119-128.

[24] Scheffler, H.-P. (2003). Precise asymptotics in Spitzer and Baum-Katz's law of large numbers: the semistable case. J. Math. Anal. Appl. 288, 285-298.

[25] Shao, Q.M. (1989). On the complete convergence for $\varrho$-mixing sequences. Acta Math. Sinica, Chinese Series 32, 377-393.

[26] Shao, Q.M. (1995). Maximal inequality for partial sums of $\varrho$-mixing sequence. Ann. Probab. 23, 948-965.

[27] Shao, Q.M. (2000). A comparison theorem on maximum ineualities between negatively associated and independent random variables. J. Theoret. Probab. 13, 343-356.

[28] Spătaru, A. (1999). Precise asymptotics in Spitzer's law of large numbers. J. Theoret. Probab. 12, 811-819.

[29] Spitzer, F. (1956). A combinatorial lemma and its applications to probability theory. Trans. Amer. Math. Soc. 82, 323-339.

[30] TAN, X.-L. AND YANG, X.-Y. (2008). A general result on precise asymptotics for linear processes of positively associated sequences. Appl. Math. J. Cinese Univ. 23, 190-196.

Allan Gut, Department of Mathematics, Uppsala University, Box 480, SE-751 06 Uppsala, Sweden; allan.gut@math.uu.se (corresponding author)

Josef Steinebach, Universität zu Köln, Mathematisches Institut, Weyertal 86-90, D-50 931 Köln, Germany; jost@math.uni-koeln.de 